\title{
EIGENFUNCTIONS OF LAPLACE OPERATORS
}

\author{
BY K. UHLENBECK
}

Communicated by M. H. Protter, May 16, 1972

Most explicit information on the eigenfunctions of a Laplace operator on a compact manifold comes from computations where a high degree of symmetry is present. In these cases, eigenspaces may be of large dimension, the zeros of the eigenfunctions are often critical points, and the eigenfunctions usually have degenerate critical points. However, these properties are all unstable under small perturbations of the metric, and are therefore rather misleading to one's intuition. From the point of view of differential topology, the best possible properties the eigenfunctions can have would be the following:

Property A. The eigenspaces are one-dimensional.

Property B. 0 is not a critical value of the eigenfunctions (so the zero or nodal set is a manifold of codimension 1).

Property $\mathrm{C}$. The eigenfunctions are Morse functions (they have nondegenerate critical points).

These properties are true for a residual set of metrics on a manifold. In the same vein we also establish similiar generic properties for bifurcations, and discuss how this approach can be used to attack the problem of invariant properties of $n$th eigenfunctions.

The idea for this work originated in some similiar work of J. Albert on eigenfunctions [3]. His methods show that the use of transversality arguments can be avoided. More general theorems and more detailed proofs will appear elsewhere [7].

The main technical tools used are the Sard-Smale theorem and the transversality theorems which follow from it, although all the proofs can be carried out directly by exhibiting open dense sets. A map $f: H \rightarrow E$ between two Banach manifolds is Fredholm if $D f_{x}: T_{x}(H) \rightarrow T_{f(x)}(E)$ has finite-dimensional kernel and cokernel, and its index is the difference in these two dimensions. A point $y \in E$ is a regular value for $f$ if $x \in f^{-1}(y)$ implies $D f_{x}$ is onto. A set of second Baire category is a residual set, and we have used generic to describe a property which is true for a residual set.

AMS 1969 subject classifications. Primary 5750; Secondary 3580, 3513.

Key words and phrases. Eigenfunctions, Laplace operators, generic, bifurcation, invariant properties. 
SARD-SMALE THEOREM [1]. Let $H$ and $E$ be separable Banach manifolds, $f: H \rightarrow E$ a $C^{k}$ Fredholm map, $k>$ index $f$. Then $\{y \in E: y$ is a regular value of $f\}$ is residual in $E$.

Transversality Theorem ([1], [5]). Let $B, H$, and $E$ be separable Banach manifolds, $\varphi: B \times H \rightarrow E$ a $C^{k}$ map, and $\varphi(b, \quad)=\varphi_{b}$ a Fredholm map of index less than $k$. Then if $y \in E$ is a regular value of $\varphi,\left\{b \in B: \varphi_{b}\right.$ has $y$ as a regular value $\}$ is residual in $B$.

Let $M$ be a compact manifold without boundary. We consider the space of $C^{k}$ metrics to be sections of a bundle

$$
\begin{aligned}
\mathscr{M}_{k}=\left\{g \in C ^ { k } \left(M, T^{*}(M)\right.\right. & \left(S T^{*}(M)\right): g(x) \cdot\left(v_{x}, v_{x}\right)>0 \\
& \text { for } \left.0 \neq v_{x} \in T_{x}(M)\right\} .
\end{aligned}
$$

For every $g \in \mathscr{M}_{k}$, we have a Laplace operator $\Delta_{g}$ which is globally defined by the equation

$$
\int_{M} g_{*}(d v, d u) d \mu_{g}=-\int_{M} v \Delta_{g} u d \mu_{g}
$$

or in local coordinates, $g=\left\{g_{i j}\right\}$, det $\left\{g_{i j}\right\}=|g|$,

$$
\Delta_{g}=\sum_{i, j}|g|^{-1} \frac{\partial}{\partial x_{i}}|g| g^{i j} \frac{\partial}{\partial x_{j}}
$$

THEOREM 1. $k>\operatorname{dim} M+1$. Then the set $\left\{g \in \mathscr{M}_{k}: \Delta_{g}\right.$ has eigenfunctions which have Properties A, B and C $\}$ is residual in $\mathscr{M}_{k}$.

Proof. Let $H_{k}(M)$ be the Sobolev space of functions on $M$ with square integrable derivatives through order $k$. Define the map

$$
\mathscr{E}: H_{k}(M) \times R \times \mathscr{M}_{k} \rightarrow H_{k-2}(M)
$$

by $\mathscr{E}(u, \lambda, g)=\Delta_{g} u+\lambda u$. This is easily seen to be a smooth map, and $\mathscr{E}_{g}=\mathscr{E}(,, g)$ is Fredholm of index 1, the eigenfunctions are those nonzero functions which lie in the inverse image of $0 \in H_{k-2}(M)$ and they are in one-dimensional eigenspaces exactly when 0 is a regular value of $\mathscr{E}_{g}$. We can show that 0 is a regular value of $\mathscr{E}$ and apply the transversality theorem to show that Property A is generic.

To show that Property B is generic, we consider the manifold of eigenfunctions

$$
Q=\left\{(g, u) \in \mathscr{M}_{k} \times H_{k}(M): \mathscr{E}(g, u, \lambda)=0 \text { and } \int_{M} u^{2} d \mu_{g}=1\right\} .
$$

$\pi: Q \rightarrow \mathscr{M}_{k}$ is the restriction of the projection. The map $\alpha: Q \times M \rightarrow R$ is a $C^{k}$ map given by $\alpha(g, u, x)=u(x)$. To show that 0 is a regular value 
of $\alpha$, we must know that the 0 set of an eigenfunction $u$ is not too bad. We use some form of the unique continuation theorem for this [4]. Once we know that 0 is a regular value of $\alpha$, we can apply a transversality theorem similiar to the one stated previously, to show that $\left\{g \in \mathscr{M}_{k}: 0\right.$ is a regular value of $\mathscr{E}_{g}$ and of $\left.\alpha_{g}=\alpha \mid \pi^{-1}(g)\right\}$ is residual in $\mathscr{M}_{k}$. When 0 is a regular value of $\mathscr{E}_{g}, \pi^{-1}(g)$ consists of isolated eigenfunctions and $\alpha \mid \pi^{-1}(g) \times M$ is just the collection of evaluation maps, so we have shown that Property B is generic. Property $C$ is similiar, but we replace $\alpha$ by a map $\beta: Q \times M \rightarrow T^{*}(M)$ given by $\beta(g, u, x)=d u(x)$.

Corollary 1. $k>\operatorname{dim} M+1$. Then the set $\left\{g \in \mathscr{M}_{k}:\right.$ Properties A, $\mathrm{B}$ and $\mathrm{C}$ are true for the first $n$ eigenfunctions $\}$ is open and dense in $\mathscr{M}_{k}$.

\section{Corollary 2. Theorem 1 is true if $k=\infty$.}

Corollary 1 is easy to prove, because it is only necessary to show that the set is open. The second corollary is implied by the first. The second theorem has a proof much like the first theorem. It might seem surprising that the curves of operators generically avoid multiple eigenspaces, but this seems to be true in general due to the jump in dimension of the eigenspaces.

THEOREM 2. Let $\mathscr{C}_{k}=\left\{G \in C^{k}\left(I, \mathscr{M}_{k}\right): G(0)=g_{1}, G(1)=g_{2}\right\}$. If $g_{1}$ and $g_{2}$ have Laplace operators which satisfy Properties A, B, C, then the set $\left\{G \in \mathscr{C}_{k}\right.$ : the eigenspaces of $\Delta_{G(t)}$ are one-dimensional, the eigenfunctions of $\Delta_{G(t)}$ have 0 as a nondegenerate critical point, and the parameterized family of nth eigenfunctions of $\Delta_{G(t)}$ are smooth functions on $M \times I$ with nondegenerate critical points and 0 as a regular value $\}$ is residual in $\mathscr{C}_{k}$.

Although progress has been made in finding properties of the eigenvalue of Laplace operators, little has been discovered recently about the eigenfunctions themselves. If $u$ is the $n$th eigenfunction of a Laplace operator, then $M-u^{-1}(0)$ must have between two and $n$ components [5]. It also follows from the maximum principle that an eigenfunction cannot take on a local maximum at a nonpositive value. The goal is to find more properties. The results of Theorem 2 suggest another approach to the problem.

Suppose we wish to find invariant properties of the $n$th eigenfunction. If we choose two different metrics, according to Theorem 1 it is not a very serious restriction to assume that their Laplace operators have Properties A, B and C. Since the set of metrics is connected, we may assume that they are connected by a curve $G \in \mathscr{C}_{k}$. Due to Theorem 2, we may assume that the eigenfunctions of $\Delta_{G(t)}$ have nice bifurcation properties. The $n$th eigenfunctions $u_{n}(x, t)$ are smooth functions on $M \times I$ with nondegenerate critical points, 0 is not a critical value, and 
when 0 is a critical value of $u_{n}(, t)$, it is nondegenerate. We now have a smooth way of passing from one set of eigenfunctions to the other. Aside from the originally mentioned restrictions on $n$th eigenfunctions, there are no obvious additional obstructions. The difficulty is to prove this.

Conjecture. Let $u(x, t)$ be a smooth function on $M \times I$ with the properties mentioned in the above paragraph. Assume in addition that $u(x, 0)$ is the $n$th eigenfunction of some Laplace operator. $u_{t}(x)=$ $u(x, t)$. If $u_{t}^{-1}$ never has a critical point at 0 of index 0 or $n$ and $M-u^{-1}(0)$ has between 2 and $n$ components, then there exists a smooth family of metrics $G(t)$ and a smooth function $r(x, t)>0$ such that $r(x, t) u(x, t)$ is the $n$th eigenfunction of $\Delta_{G(t)}$.

REMARK. This could be stated in terms of a cobordism of the nodal set. This conjecture is true rather obviously when 0 is not a critical value of $u_{t}(x)$. If the conjecture is not true, one would like to identify more obstructions.

It is very possible that there are a greater number of invariants for the $n$th eigenfunction if only conformal changes of the metric are allowed. Conformal changes are particularly easy to work with.

After the structure of one eigenfunction is examined, it is possible to consider the relationships between two eigenfunctions. The following proposition is an exercise which can be proven from the information in Courant-Hilbert. It is certainly possible to get a generic form for the intersection of the nodal sets.

Proposition. Let $u(x)$ and $v(x)$ be nonconstant eigenfunctions. If $M-u^{-1}(0)$ and $M-v^{-1}(0)$ have only two components, then $u^{-1}(0) \cap$ $v^{-1}(0) \neq \varnothing$.

\section{REFERENCES}

1. R. Abraham, Transversality in manifolds of mappings, Bull. Amer. Math. Soc. 69 (1963), 470-474. MR 26 \#6982.

2. S. Agmon, Lectures on elliptic boundary value problems, Van Nostrand, Princeton, N.J., 1965. MR 31 \# 2504.

3. J. H. Albert, Nodal and critical sets for eigenfunctions of elliptic operators, Proc. Sympos. Pure Math., vol. 23, Amer. Math. Soc., Providence, R.I., 1973, pp. 71-78.

4. N. Aronszajn, A unique continuation theorem for solutions of elliptic partial differential equations or inequalitites of second order, J. Math. Pures Appl. (9) 36 (1957), 235-249. MR 19, 1056.

5. R. Courant and D. Hilbert, Methods of mathematical physics. Vol. 1, Interscience, New York, 1953. MR 16, 426.

6. F. Quinn, Transversal approximation on Banach manifolds, Proc. Sympos. Pure Math., vol. 15, Amer. Math. Soc., Providence, R.I., 1970, pp. 213-222. MR 41 \#9304.

7. K. Uhlenbeck, Generic properties of eigenfunctions (in preparation).

Department of Mathematics, University of Illinois at Urbana-Champaign, URBANA, ILLINOIS 61801 